\title{
Serum is an indispensable factor in the maintenance of the biological characteristics of sweat gland cells
}

\author{
QING SUN ${ }^{1,2^{*}}$, XIAO-MEI DENG ${ }^{2 *}$, YUN-LIANG WANG ${ }^{3 *}$, YUN-FANG ZHEN ${ }^{4}$, FANG LI $^{2,5}$, \\ RUI-HUA CHEN ${ }^{1,2}$, HAN-SI LIANG ${ }^{1,2}$, FENG ZHANG ${ }^{2,6}$, MING-DE QIN ${ }^{2,6}$ and XUE-GUANG ZHANG ${ }^{1,2}$ \\ ${ }^{1}$ Jiangsu Institute of Clinical Immunology, The First Affiliated Hospital of Soochow University; \\ ${ }^{2}$ The Stem Cell and Biomedical Material Key Laboratory of Jiangsu; ${ }^{3}$ Department of General Surgery, \\ The First Affiliated Hospital of Soochow University, Soochow University, Suzhou, Jiangsu 215007; ${ }^{4}$ Institute of \\ Pediatrics, Children's Hospital of Soochow University, Suzhou, Jiangsu 215005; ${ }^{5}$ Department of Human Anatomy, \\ Histology and Embryology, School of Biology and Basic Medical Sciences; ${ }^{6}$ Department of Immunology, \\ School of Biology and Basic Medical Sciences, Soochow University, Suzhou, Jiangsu 215123, P.R. China
}

Received March 19, 2016; Accepted May 8, 2017

DOI: $10.3892 / \mathrm{mmr} .2017 .6909$

\begin{abstract}
The tolerance of sweat gland cells for in vitro amplification and subcultivation is low as they are somatic cells. The present study aimed to formulate an optimal medium for the culture of human eccrine sweat gland cells (HESGCs) and to establish a method for induction of HESGCs proliferation, whilst maintaining the characteristics of sweat gland cells. HESGCs cultured in sweat gland (SG):keratinocyte growth medium-2 (KGM-2) (1:1) medium had a higher proliferation rate and a stable morphology compared with cells cultured in SG and KGM-2 medium only. Reverse transcription-quantitative polymerase chain reaction indicated that cells cultured in the SG:KGM-2 (1:1) medium exhibited higher expression levels of $\alpha$-smooth muscle actin, keratin (K)77, carcinoembryonic antigen, $\mathrm{K} 8, \mathrm{~K} 18$, ectodysplasin A receptor, c-Myc, Kruppel-like factor 4 and octamer-binding transcription factor 4 compared with cells cultured in SG only or KGM-2 only medium. Three-dimensional culture analysis revealed that HESGCs cultured in SG:KGM-2 1:1 medium differentiated into sweat gland-like structures, whereas cells cultured in KGM-2 only medium underwent cornification. The present study also determined that the maintenance of the biological characteristics of HESGCs occurred due to the presence of fetal bovine serum (FBS). Cells cultured in medium without FBS
\end{abstract}

Correspondence to: Dr Xue-Guang Zhang or Dr Ming-De Qin, The Stem Cell and Biomedical Material Key Laboratory of Jiangsu, Soochow University, 708 RenMin Road, Suzhou, Jiangsu 215007, P.R. China

E-mail: xueguangzh@126.com

E-mail: leopardxq@hotmail.com

*Contributed equally

Key words: sweat gland cells, culture media, serum, keratinocytes, tissue engineering differentiated into keratinocytes. Therefore, the SG:KGM-2 (1:1) medium may be a suitable culture medium for HESGCs. In conclusion, this mixed medium is a valuable compound and should be considered to be a potential supplemental medium for HESGCs.

\section{Introduction}

Sweat glands are major skin structures distributed across human skin that are vital for temperature regulation and electrolyte balance $(1,2)$. An increasing number of patients with large area burns suffer from sweat gland defects, which severely affect their quality of life (3). Additionally, human eccrine sweat gland cells (HESGCs) have been reported to be capable of repair of wounded human skin $(4,5)$. Therefore, increasing the availability of therapeutic sweat glands is required for future clinical studies. However, HESGCs have a limited proliferation capacity in the currently used culture conditions $(6,7)$. Previous studies have determined that bone marrow mesenchymal and hair follicle stem cells may be induced to differentiate into sweat gland cells in order to produce more HESGCs in vitro $(8,9)$. However, previous studies have not compared the efficacy of different basal media for the culture of sweat gland cells. Therefore, it is necessary to improve knowledge of the current culture media to be used in future studies.

As presented in Table I, there are two major categories of media presently used to cultivate HESGCs, media with and without serum (10-18). Sweat gland (SG) medium was the most commonly used medium, containing fetal bovine serum (FBS). Similarly, keratinocyte growth medium-2 (KGM-2) is a commonly used serum-free medium.

The present study revealed that HESGCs cultured in SG medium maintained the biological characteristics of HESGCs; however, they demonstrated slow cell growth. HESGCs cultured in KGM-2 medium demonstrated increased proliferation rates; however, the cells gradually lost the biological characteristics of sweat gland cells. The aforementioned findings of the present 
study implied that competitive culture conditions are required for HESGCs.

The present study aimed to mix the two media (SG and KGM-2) in order to increase cell growth and maintain the biological characteristics of HESGCs. The efficiency of the three media types was compared and the mixed medium (SG:KGM-2 medium 1:1) was identified to provide the most suitable HESGC culture conditions on the basis of proliferation ability and maintenance of sweat gland cell biological characteristics. Additionally, the present study identified that the loss of the sweat gland cell biological characteristics in serum-free medium occurred as the sweat gland cells rapidly differentiated into keratinocytes without serum.

\section{Materials and methods}

Human skin tissues. A total of 12 infant polydactyl skin samples were collected between January 2015 and January 2016 from male children aged 1-3 years old who had a supernumerary sixth finger. The samples are used for present study with the written informed consent of patients' parents and the written approval was obtained from the ethical review board of Children's Hospital Affiliated with Soochow University (Suzhou, China). These skin samples were used for the isolation of sweat gland cells. All methods were performed in accordance with the approved guidelines. All experimental protocols were approved by Soochow University. Copies of the written consent provided by the subjects along with the written approval from the review board were kept in the Children's Hospital Affiliated with Soochow University ethical review board office. All experimental procedures using skin samples in the present study were reviewed and approved by the ethics committee.

Isolation and culture of primary human sweat gland cells. Following the removal of subcutaneous tissue, the skin samples were minced into smaller pieces with sharp scissors in phosphate buffered saline (PBS), then epidermal and dermal tissues were separated using dispase II $(1 \mathrm{mg} / \mathrm{ml}$; Roche Applied Science, Penzberg, Germany) at $4^{\circ} \mathrm{C}$ for $\sim 18 \mathrm{~h}$. The dermal tissue was digested with collagenase type IV $(2.5 \mathrm{mg} / \mathrm{ml}$; Sigma-Aldrich, Merck Millipore, Darmstadt, Germany) in an incubator at $37^{\circ} \mathrm{C}$ for $1 \mathrm{~h}$. Sweat gland tissues became accessible following digestion and were collected using a Transferpettor under an ultraviolet-sterilized phase-contrast inverted microscope at magnification x40.

The collected sweat gland tissues were seeded into a 6-well plate (Corning, Inc., Corning, NY, USA) in SG medium at a density of $\sim 120$ tissues/well. The SG medium contained Dulbecco's modified Eagle's medium-F12 (DMEM-F12, Gibco; Thermo Fisher Scientific, Inc., Waltham, MA, USA) with $4.5 \mathrm{~g} / \mathrm{l}$ glucose, supplemented with $10 \%$ FBS (Hyclone; GE Healthcare Life Sciences, Logan, UT, USA), 1\% penicillin streptomycin (Gibco; Thermo Fisher Scientific, Inc.), 2 mM L-glutamine (Gibco; Thermo Fisher Scientific, Inc.), 10x insulin-transferrin sodium selenite solution (ITS, Gibco; Thermo Fisher Scientific, Inc.), 2 nM triiodothyronine (Sigma-Aldrich, Merck Millipore), $0.4 \mathrm{mg} / \mathrm{ml}$ hydrocortisone (Sigma-Aldrich, Merck Millipore), and $10 \mathrm{ng} / \mathrm{ml}$ human recombinant epidermal growth factor (R\&D Systems, Inc.,
Minneapolis, MN, USA) (19). When the sweat gland cells migrated from the tissues after $\sim 5$ days in the SG medium, three different culture media [SG, KGM-2 (Lonza Group, Ltd., Basel, Switzerland) and SG:KGM-2 (1:1)] were added in order to observe cellular proliferation and phenotypes.

$R N A$ isolation and reverse transcription-quantitative polymerase chain reaction $(R T-q P C R)$. Total RNA was isolated from the HESGCs cultured in the different media using the RNeasy Mini extraction kit (Qiagen, Inc., Valencia, CA, USA) according to the manufacturer's protocol. A total of $1 \mu \mathrm{g}$ RNA was used for reverse transcription (Qiagen, Inc., Valencia, CA, USA). For the qPCR assays, the primer mixes were loaded in duplicate wells in 96-well plates and then reactions were performed following the addition of SYBR Green PCR Master mix (Thermo Scientific, Inc., Waltham, MA, USA) and $1 \mu \mathrm{g}$ (final) cDNA. The experiment was repeated for three times. After pre-denaturation at $95^{\circ} \mathrm{C}$ for $5 \mathrm{~min}, 30 \mathrm{sec}$ at $95^{\circ} \mathrm{C}$ cooling to $65^{\circ} \mathrm{C}$ for $45 \mathrm{sec}$ for 40 cycles (Bio-Rad Laboratories, Inc., Hercules, CA, USA). As an internal control, GAPDH levels were quantified with target genes in parallel. Normalization and fold-changes were calculated using the $2^{-\Delta \Delta \mathrm{Cq}}$ method (20), and the primers used are presented in Table II.

Three-dimensional culture of sweat gland cells derived from different media. Collagen I from bovine tendon (Gibco; Thermo Fisher Scientific, Inc.) and Matrigel (BD Biosciences, Franklin Lakes, NJ, USA) were used to establish a dermal layer on a polycarbonate membrane. The acellular collagen matrix layers were allowed to stand at room temperature for $\sim 20$ min and solidify, subsequently a cellular collagen matrix layer mixed with $3.3 \times 10^{4}$ fibroblasts and $1 \times 10^{5}$ sweat gland cells was added. The mixture was incubated for $1 \mathrm{~h}$ at $37^{\circ} \mathrm{C}$ in order to allow it to solidify. When the mixtures were solidified, EpiLife medium (Gibco; Thermo Fisher Scientific, Inc.) was used to cover the surface of the gels and this medium was changed every two days. The gels were subsequently used for experiments after 21 days of culture in an incubator at $37^{\circ} \mathrm{C}$.

Sweat gland-like structure counting standards. A protocol to quantify the number of sweat gland-like structures that formed in the gels was designed. A total of 3 sections were selected randomly with an interval of at least $100 \mu \mathrm{m}$ to avoid the same structure being counted repeatedly. The counting standards were as follows: Sweat gland-like structures formed by sweat gland cells in different culture media were defined as structures composed of 6-15 cells in one region, which is similar to the transverse section of normal sweat gland tissue. The number of structures was confirmed under an optical microscope (magnification, x10 and x40).

Isolation and culture of primary human fibroblasts. The skin samples obtained from the patients' mentioned earlier were minced into smaller pieces with sharp scissors in phosphate buffered saline (PBS), then epidermal and dermal tissues were separated using dispase II (1 mg/ml; Roche Diagnostics $\mathrm{GmbH}$, Mannheim, Germany) at $4^{\circ} \mathrm{C}$ for $\sim 18 \mathrm{~h}$. The dermal tissue was digested with trypsin (0.25\% trypsin, $0.1 \%$ EDTA; Beyotime Institute of Biotechnology, JiangSu, China) in an incubator at $37^{\circ} \mathrm{C}$ for $10 \mathrm{~min}$. Fibroblasts were cultured in DMEM (Thermo 
Table I. Supplements for media with or without serum.

\begin{tabular}{|c|c|c|c|c|c|c|c|}
\hline \multirow[b]{2}{*}{ Factor } & \multicolumn{4}{|c|}{ Media with serum } & \multicolumn{3}{|c|}{ Media without serum } \\
\hline & SG & FAD & Williums E & Rheinwald and green & KFSM & EpiLife & KGM-1/2 \\
\hline FBS & + & + & + & + & - & - & - \\
\hline BPE & - & - & - & - & + & + & + \\
\hline hEGF & + & + & + & + & + & + & + \\
\hline Insulin & - & + & - & + & + & + & + \\
\hline Hydrocortisone & + & + & + & + & + & + & + \\
\hline Transferrin & - & - & - & - & + & + & + \\
\hline Epinephrine & - & - & - & - & + & - & + \\
\hline GA-1000 & - & - & - & - & - & + & + \\
\hline Triiodothyronine & + & + & - & + & - & - & - \\
\hline Insulin-transferrin-selenium & + & - & + & - & - & - & - \\
\hline Adenine & - & + & - & + & - & - & - \\
\hline Cholera toxin & - & + & - & + & - & - & - \\
\hline Reference & $(10)$ & $(11)$ & $(12,13)$ & (14) & $(15,16)$ & $(17)$ & (18) \\
\hline
\end{tabular}

FBS, fetal bovine serum; BPE, bovine pituitary extract; hEGF, human epidermal growth factor; FAD, flavin adenine dinucleotide; SG, sweat gland medium; KGM-2, keratinocyte growth medium-2.

Fisher Scientific, Inc.) with $4.5 \mathrm{~g} / 1$ glucose, supplemented with $10 \%$ FBS (Hyclone; GE Healthcare Life Sciences), $1 \%$ penicillin streptomycin (Gibco; Thermo Fisher Scientific, Inc.), 2 mM L-glutamine (Gibco; Thermo Fisher Scientific, Inc.). Approximately $95 \%$ of the non-adherent cells were removed after $24 \mathrm{~h}$, and the culture media was replaced every day. Cells were passaged by trypsinization $(0.25 \%$ trypsin, $0.1 \%$ EDTA) and expanded serially with a split ratio of $1: 3$ at $70 \%$ confluence in 2 or 3 days.

Three-dimensional air-liquid interface tissue culture. Collagen I from bovine tendon, populated with fibroblasts, was used for establishing successive layers of acellular and cellular collagen on a polycarbonate membrane. The collagen I was prepared as aforementioned. After $\sim 7$ days, $1 \times 10^{5}$ sweat gland cells from the three different media cultures were added to the center of the collagen gel and incubated for $1 \mathrm{~h}$ at $37^{\circ} \mathrm{C}$ to allow the sweat gland cells to fully adhere. Cells were exposed to the air-liquid interface for 8 days in EpiLife medium.

Histochemistry analysis. Cells were fixed with 4\% paraformaldehyde, dehydrated, and embedded in paraffin for hematoxylin $\&$ eosin (H\&E) staining. The samples were observed under an optical microscope, where 10 different views, selected at random, were examined at magnifications x10, x20 and x40 (Leica Microsystems, Inc., Buffalo Grove, IL, USA).

Statistical analysis. Data are presented as the mean \pm standard deviation and were acquired following at least 3 independent experiments prior to being analyzed using Prism 5 software (Graph Pad Software, San Diego, CA, USA). The qPCR data are presented as the fold-change in gene expression normalized to GAPDH (used as a reference gene) using the $2^{-\Delta \Delta C q}$ method. Differences between each group were analyzed using one-way analysis of variance followed by a Tukey's HSD post-hoc test. $\mathrm{P}<0.05$ was considered to indicate a statistically significant difference.

\section{Results}

Biological characteristics of sweat gland cells cultured in different media. Different cell morphologies resulted from sweat gland cell culture in different media. HESGCs cultured in SG medium and SG:KGM-2 (1:1) medium maintained an HESGC-like morphology. A higher number of fibroblasts survived in the SG medium compared with the other media. HESGCs cultured in KGM-2 medium had a clear boundary, significant cell interval and typical keratinocyte-like morphology (Fig. 1A) as previously described (21). The cells were subcultured three times in succession, at passage 0, 1 and 2. HESGCs that were cultured in SG:KGM-2 (1:1) medium had higher proliferation compared with those cultured in SG medium or KGM-2 medium alone (Fig. 1B). RT-qPCR was used to analyze the mRNA expression levels of marker genes of HESGCs, including $\alpha$ smooth muscle actin $(\alpha-S M A)$, keratin (K)77, carcinoembryonic antigen (CEA), K8, K18 and ectodysplasin A (EDA) and stem cell markers, such as c-Myc, Kruppel-like factor 4 (Klf-4) and octamer-binding transcription factor 4 (Oct-4). The cells cultured in SG:KGM-2 (1:1) medium had higher mRNA expression levels of HESGCs gland markers ( $\alpha$-SMA, CEA, K8, K18 and EDA) and stem cell markers (c-Myc, Klf-4 and Oct-4) compared with cells cultured in SG medium or KGM-2 medium only. The expression levels of the markers (CEA, K8, K18, EDA, c-Myc, and Oct-4) in the SG:KGM-2 (1:1) medium-cultured cells did not differ significantly from the expression levels of the markers in normal sweat gland tissue. However, the expression level of K77, a marker of the sweat gland duct, was very low in the 
Table II. Primers for reverse transcription-quantitative polymerase chain reaction.

\begin{tabular}{lcll}
\hline Gene name & Amplicon length (bp) & \multicolumn{1}{c}{ Forward (5'-3') } & \multicolumn{1}{c}{ Reverse (5'-3') } \\
\hline$\alpha$-SMA & 122 & CTATGAGGGCTATGCCTTGCC & GCTCAGCAGTAGTAACGAAGGA \\
K77 & 105 & TCTCAGTCCGCGTTTAGTTCA & TCGAGCATAACACACAGAACC \\
CEA & 138 & AAGAAATGACGCAAGAGCCTATG & CCCGAAAGGTAAGACGAGTCTG \\
K8 & 150 & TCCTCAGGCAGCTATATGAAGAG & GGTTGGCAATATCCTCGTACTGT \\
K18 & 171 & TCGCAAATACTGTGGACAATGC & GCAGTCGTGTGATATTGGTGT \\
EDA & 155 & AGATGGCCCAGTTAAAAACAAGA & CAGGTGGTCCCATAACAGTTG \\
EDAR & 121 & CAGCCCGAGCGGAATACTC & CCGTAGCCACAGGACAGGTA \\
c-Myc & 216 & TCAAGAGGCGAACACACAACGTCT & GTTCTCGTCGTTTCCGCAACAAGT \\
Klf-4 & 139 & CGGACATCAACGACGTGAG & GACGCCTTCAGCACGAACT \\
Oct-4 & 164 & CTTGAATCCCGAATGGAAAGGG & GTGTATATCCCAGGGTGATCCTC \\
K5 & 75 & ATCTCTGAGATGAACCGGATGATC & CAGATTGGCGCACTGTTCTT \\
K14 & 80 & GGCCTGCTGAGATCAAAGACTAC & CACTGTGGCTGTGAGAATCTTGTT \\
GAPDH & 197 & GGAGCGAGATCCCTCCAAAAT & GGCTGTTGTCATACTTCTCATGG
\end{tabular}

$\alpha$-SMA, $\alpha$ smooth muscle actin; K77/8/18/5/14, keratin 77/8/18/5/14; CEA, carcinoembryonic antigen; EDA, ectodysplasin A; EDAR, EDA receptor; Klf-4, Kruppel-like factor 4; Oct-4, octamer-binding transcription factor 4.

three medium groups. The lack of K77 expression suggested that the majority of the sweat gland cells cultured in vitro in the present study were derived from the secretory portion of the gland. These findings indicate that the SG:KGM-2 (1:1) medium is more suitable for culturing HESGCs compared with SG or KGM-2 medium primarily due to the improved maintenance of the HESGCs morphology (Fig. 1C).

HESGCs cultured in different media form sweat gland-like structures. In order to determine whether HESGCs may differentiate into sweat gland-like structures in vitro, the present study compared the cell structures resulting from three-dimensional culture conditions. The sweat gland cells cultured in SG medium and SG:KGM-2 (1:1) medium formed sweat gland-like structures, whereas sweat gland cells cultured in KGM-2 only medium did not differentiate into sweat gland-like structures (Fig. 2A). In addition to the formation of sweat gland-like structures, the number of sweat gland-like structures formed during the culture in different media was also monitored. HESGCs cultured in SG:KGM-2 (1:1) medium formed a significantly greater number of sweat gland-like structures compared with cells cultured in SG medium only (Fig. 2B). However, no sweat gland-like structures were formed in the KGM-2 only medium. In summary, the SG:KGM-2 (1:1) medium was suitable for the culture of sweat gland cells and the formation of sweat gland-like structures.

HESGCs cultured in KGM-2 medium differentiate into epithelium. In order to determine why HESGCs cultured in KGM-2 medium did not differentiate into sweat gland-like structures, the mRNA expression of the keratinocyte markers K14 and K5 was determined. K14 and K5 expression levels in the HESGCs cultured in KGM-2 only medium were higher compared with the remaining groups (Fig. 3A). Subsequently, the air-liquid interface system was used to culture the cells derived from the three different media groups. After 8 days of air-liquid differentiation, H\&E staining revealed that cells cultured in KGM-2 medium exhibited a stratified epithelium with basal layer, stratum spinosum, stratum granulosum and stratum corneum observed as superposed layers of dead, squamous, enucleated cells. However, HESGCs cultured in SG medium and SG:KGM-2 (1:1) medium did not form completely stratified epithelia (Fig. 3B). These findings demonstrated that HESGCs cultured in KGM-2 medium were unable to differentiate into a sweat gland-like structure; however, sweat gland cells cultured in KGM-2 only medium rapidly differentiate into keratinocytes.

Serum allowed the survival of fibroblasts and the maintenance of HESGCs biological characteristics. From the various components (data not shown) of the media used in the present study, the presence or absence of serum had the greatest impact (Table I). In order to determine whether the serum was the primary factor supporting the maintenance of the desired HESGC biological characteristics and functions and to determine whether media with or without serum would lead to sweat gland cells rapidly differentiating into keratinocytes, HESGCs were cultured in four different media: i) SG medium; ii) SG medium without FBS; iii) KGM-2 medium; and iv) KGM-2 medium with FBS. Morphology of cells in the different media at 2, 4 and 6 days are presented in Fig. 4A. Due to the presence of serum in the SG medium and the KGM-2 medium with FBS, the sweat gland cells were able to maintain their morphology. However, cells cultured in the media without FBS, such as the SG medium without FBS and the KGM-2 medium, differentiated into keratinocyte-like cells. In order to determine whether these 4 groups of cells may form sweat gland-like structures, they were transferred into three-dimensional culture systems. H\&E staining revealed that cells cultured in the media without FBS did not have the ability to form sweat gland-like structures. In 

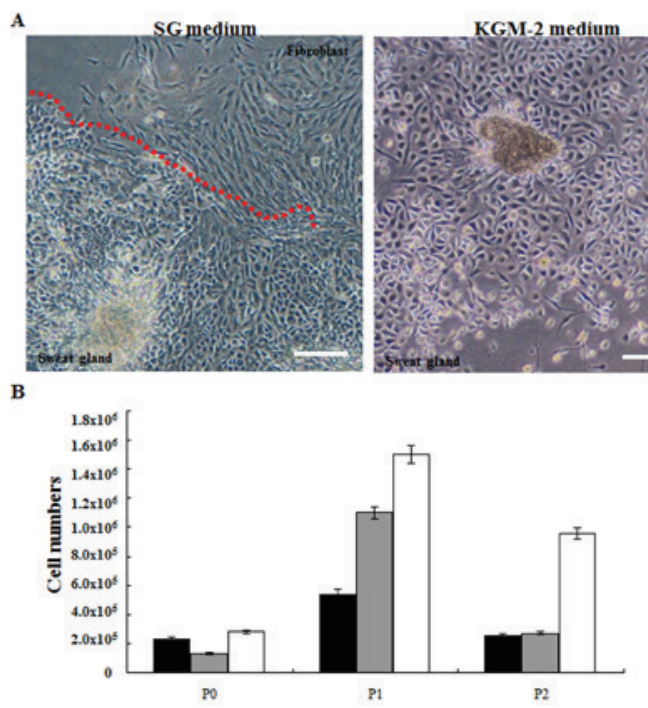

KGM-2 medium

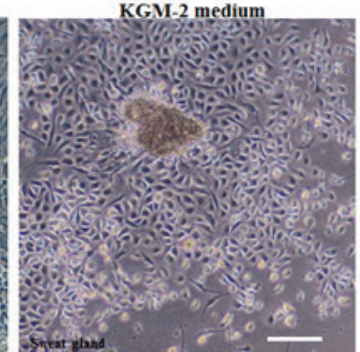

- SG medium

口KGM-2 medium

$\square$ SG : KGM-2 (1:1) medium

$S G: K G M-2(1: 1)$ medium
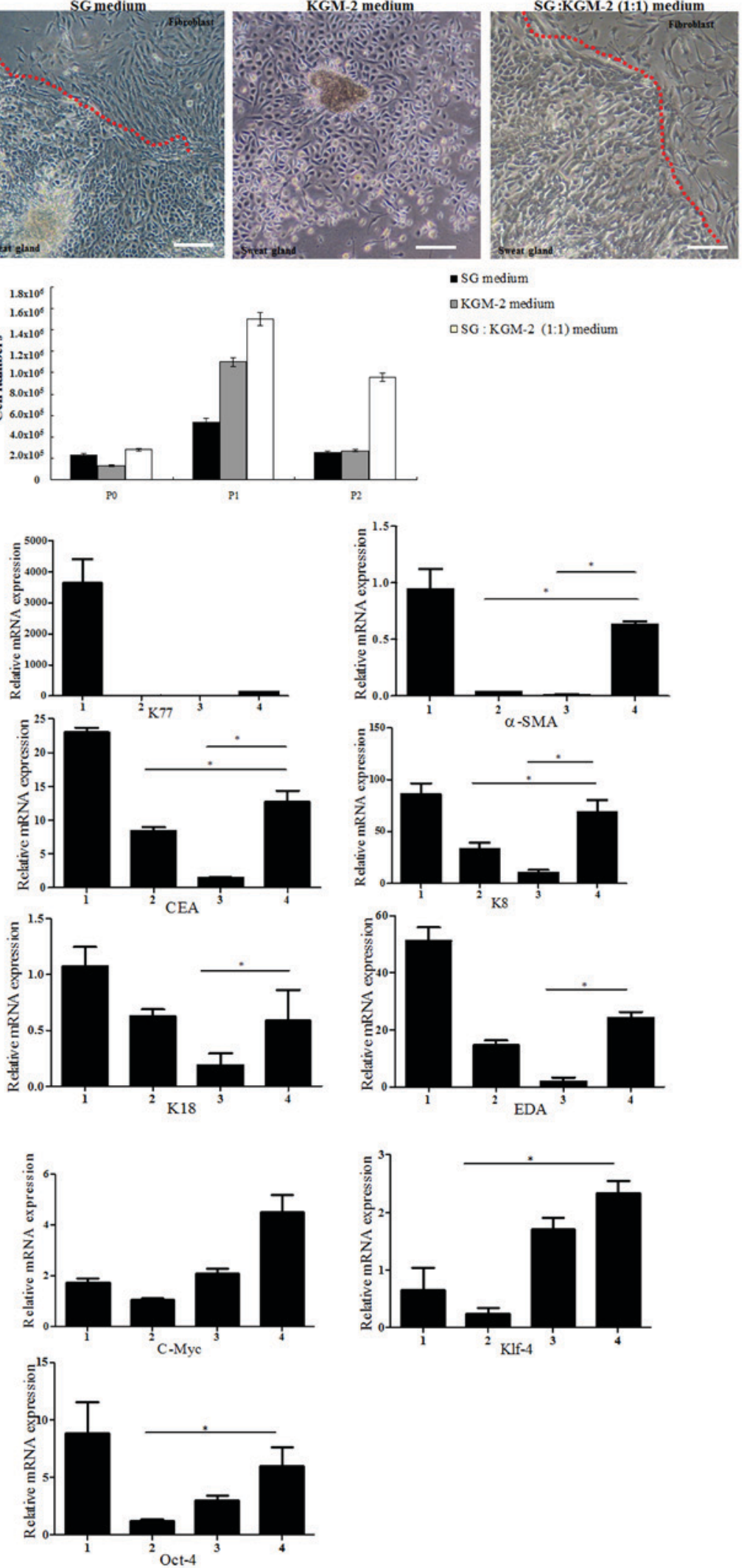

Figure 1. (A) Morphology of sweat gland cells cultured in three different media. Cells cultured in KGM-2 medium had typical epithelial morphology, whereas cells cultured in SG:KGM-2 (1:1) medium exhibited the same morphology as cells cultured in normal SG medium. The red dotted line indicates the separation between sweat gland cells and fibroblasts. (B) Following subculture of sweat gland cells to passage 3, cells cultured in the mixed medium (SG:KGM-2 1:1 medium) demonstrated a higher proliferation rate compared with cells cultured in the remaining two media. (C) mRNA expression levels of sweat gland cell markers ( $\alpha$-SMA, K77, CEA, K8, K18 and EDA) and stem cell markers (c-Myc, Klf-4 and Oct-4) were quantified in sweat gland cells cultured in three different media. The sweat gland tissues were used as positive control. Data are presented as the mean \pm standard deviation of three independent experiments. "P<0.05. Scale bar, $40 \mu \mathrm{m}$. SG, sweat gland medium; KGM-2, keratinocyte growth medium-2; 1, sweat gland tissue; 2, SG medium; 3, KGM-2 medium; 4, SG:KGM-2 (1:1) medium; $\alpha$-SMA, $\alpha$ smooth muscle actin; K77/8/18, keratin 77/8/18; CEA, carcinoembryonic antigen; EDA, ectodysplasin A; Klf-4, Kruppel-like factor 4; Oct-4, octamer-binding transcription factor 4 . 


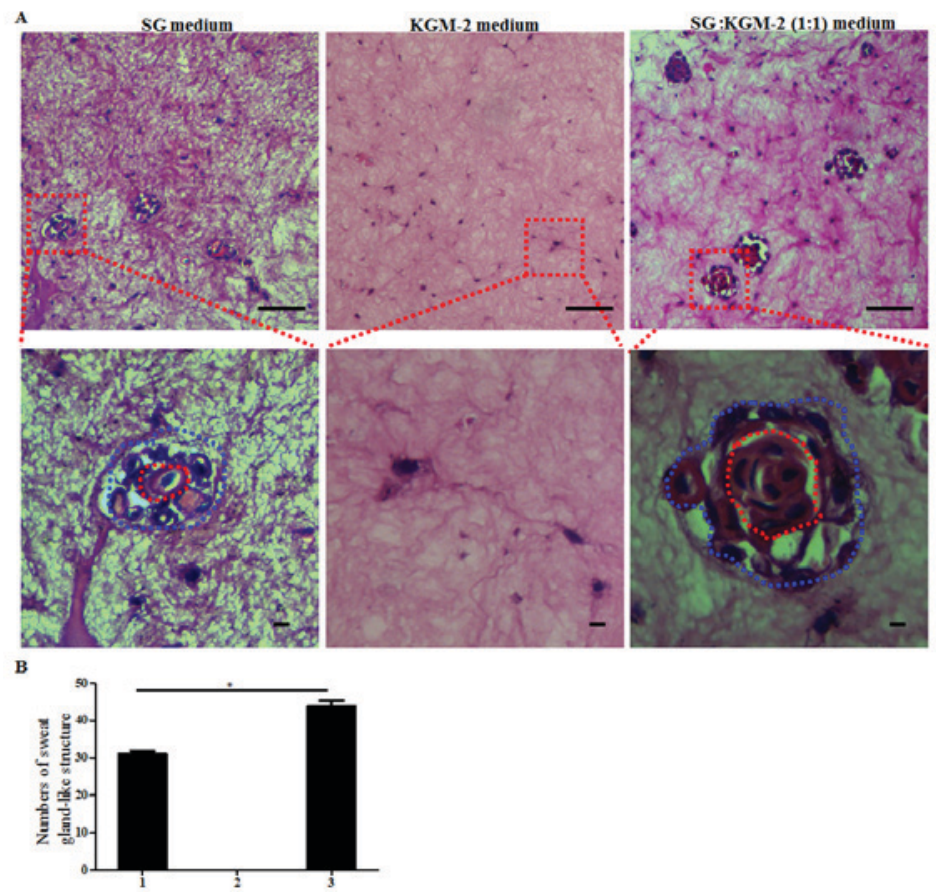

Figure 2. HESGCs cultured in recommended medium may differentiate into sweat gland-like structures in vitro. (A) Hematoxylin and eosin staining images of sweat gland-like structures formed by cells were cultured in normal SG, KGM-2 and SG:KGM-2 (1:1) medium. HESGCs cultured in SG medium and SG:KGM-2 (1:1) medium were able to form sweat gland-like structures, whereas cells cultured in KGM-2 medium did not. Blue dashed circle indicates the shape of the tubule-like structure. Red dashed circle, indicates the lumen of the tubule-like structure. (B) Number of sweat gland-like structures derived from cells cultured in three media. HESGCs cultured in the SG:KGM-2 (1:1) medium formed more sweat gland-like structures compared with cells cultured in SG medium and KGM-2 medium (n=4). 1, SG medium; 2, KGM-2 medium; 3, SG:KGM-2 (1:1) medium. "P<0.05. Scale bar, $20 \mu$ m. SG, sweat gland medium; KGM-2, keratinocyte growth medium-2; HESGCs, human eccrine sweat gland cells.
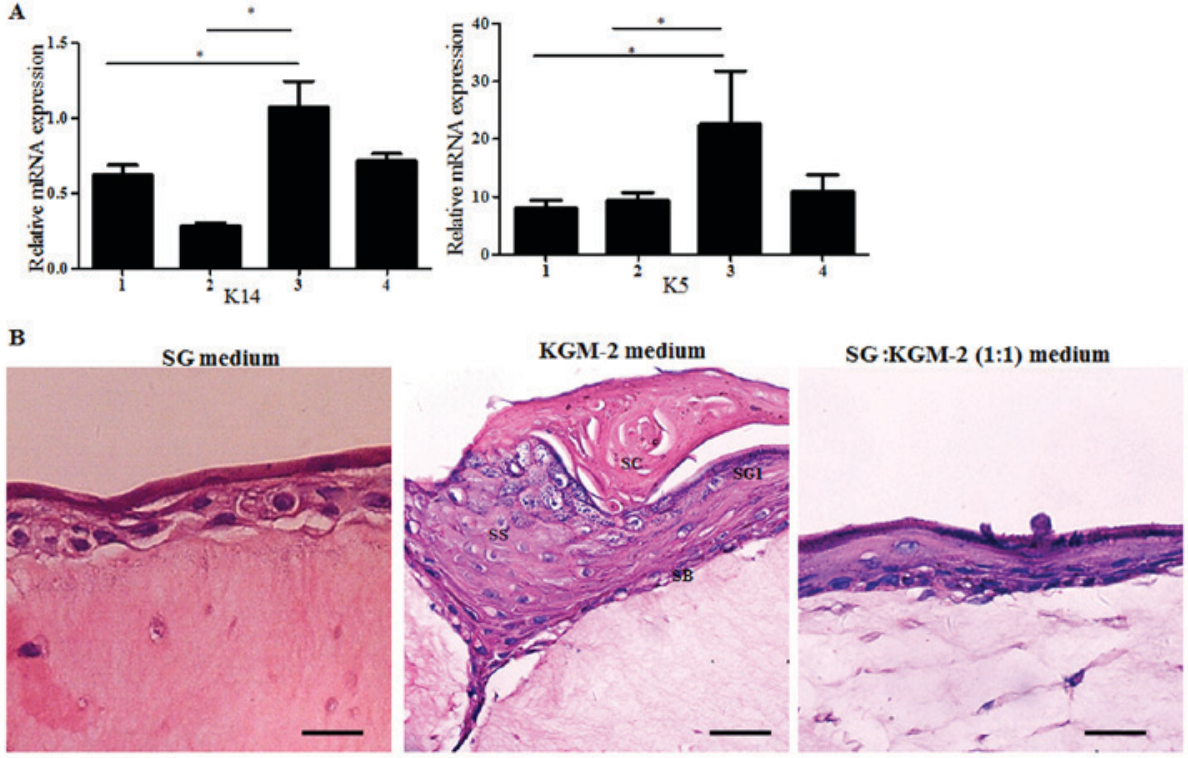

Figure 3. Sweat gland cells cultured in KGM-2 medium differentiated into keratinocytes. (A) mRNA expression of keratinocyte markers (K14, K5) in sweat gland cells cultured in three different media. The sweat gland tissues were used as negative controls. Cells cultured in KGM-2 medium had highest K5 mRNA expression among the cells cultured in the three media. Data are presented as the mean \pm standard deviation of three independent experiments. ${ }^{*} \mathrm{P}<0.05$. (B) Hematoxylin and eosin staining images of epidermis constructed by sweat gland cells. Cells cultured in KGM-2 medium displayed a well-organized, multilayer epithelium that included all morphological layers (SC, SG1, SS, and SB); however, human eccrine sweat gland cells cultured in SG medium and SG: KGM-2 (1:1) medium did not differentiate into epidermis. Scale bar, $20 \mu \mathrm{m}$. SG, sweat gland medium; KGM-2, keratinocyte growth medium-2; 1, sweat gland tissue; 2, SG medium; 3, KGM-2 medium; 4, SG:KGM-2 (1:1) medium; SC, stratum corneum; SG1, stratum granulosum; SS, stratum spinosum; SB, stratum basal; K5/14, keratin 5/14.

addition, cells cultured in the media with FBS were able to form sweat gland-like structures, this is partly due to FBS allowing for the survival of fibroblasts, which provide essential factors such as transforming growth factor- $\beta$ (TGF- $\beta$ ) 

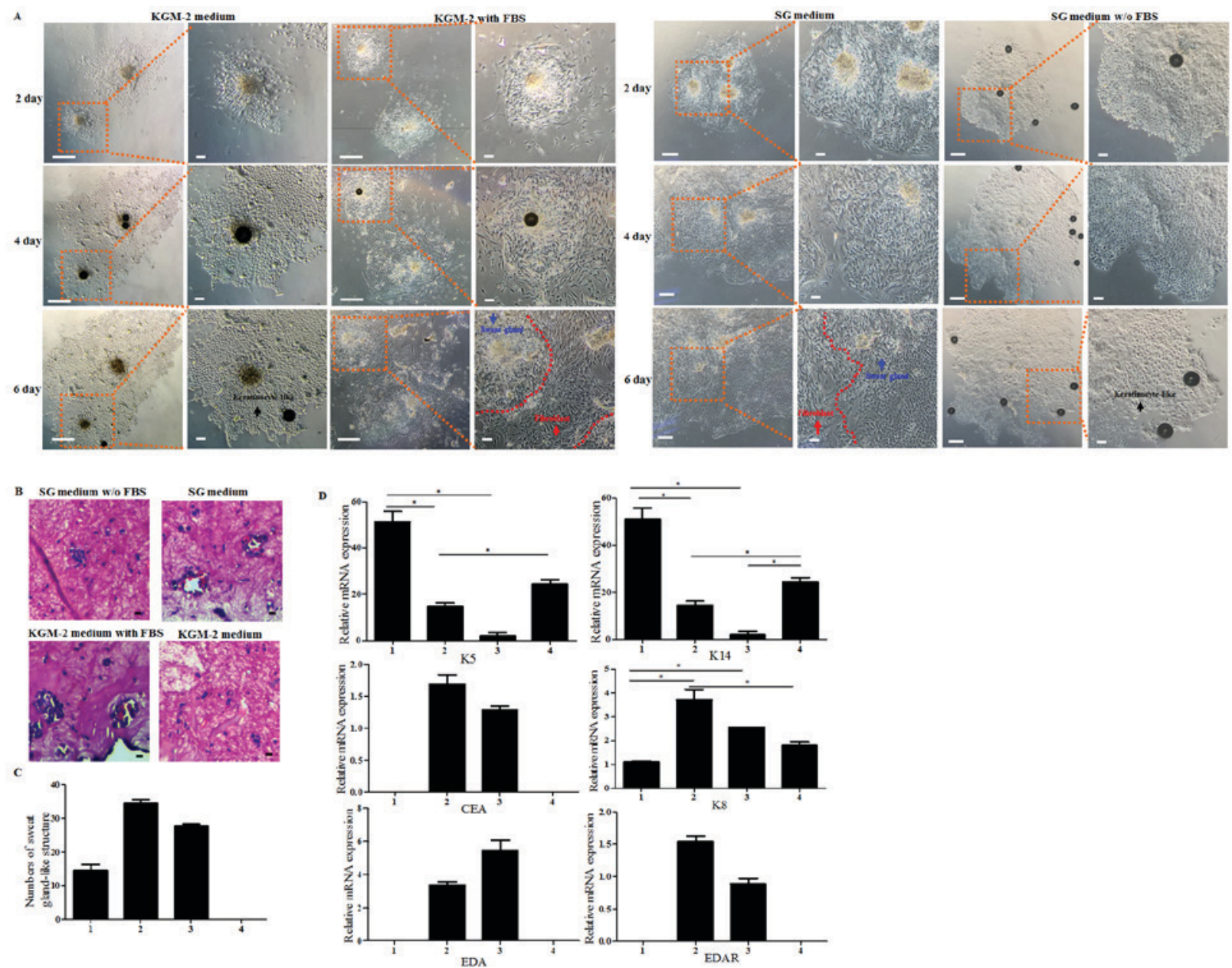

Figure 4. Serum is an indispensable factor for the maintenance of HESGCs morphology and biological characteristics. (A) Representative images of HESGCs cultured in four different media, including SG medium, SG medium without FBS, KGM-2 medium, and KGM-2 medium with FBS, at days 2, 4, 6. Fibroblasts continued to exist in the media with FBS, SG medium and KGM-2 medium with FBS. Red arrow indicates fibroblasts. Blue arrow indicates sweat gland cells. Black arrow indicates keratinocyte-like cells. Sweat gland cells and fibroblasts were distinguished by the red dotted line. (B) Hematoxylin and eosin staining images of sweat gland-like structures formed in gels by cells under four different culture conditions, including SG without FBS, SG medium, KGM-2 with FBS, and KGM-2 medium. Cells cultured in medium with FBS formed more sweat gland-like structures. (C) Numbers of sweat gland-like structures derived from cells cultured in four different media. Cells cultured in SG medium and KGM-2 with FBS medium formed more sweat gland-like structures, whereas cells cultured in KGM-2 medium did not form this type of structure due to cornification (n=3). 1, SG medium without FBS; 2, SG medium; 3, KGM-2 with FBS; 4, KGM-2 medium. (D) mRNA expression levels of sweat gland cell markers (CEA, K8, EDA, and EDAR) and keratinocyte markers (K5 and K14) in media without FBS (KGM-2 and SG medium without FBS) and media with FBS (SG medium and KGM-2 medium with FBS). 1, KGM-2 medium; 2 , KGM-2 with FBS; 3, SG medium; 4, SG medium without FBS. Data are presented as the mean \pm standard deviation of three independent experiments. "P $<0.05$. Scale bar, $10 \mu \mathrm{m}$. SG, sweat gland medium; KGM-2, keratinocyte growth medium-2; K5/14/8, keratin 5/14/8; CEA, carcinoembryonic antigen; EDA, ectodysplasin A; EDAR, EDA receptor.

and platelet-derived growth factor (PDGF) for maintaining the desired cellular biological characteristics of HESGCs (Fig. 4B). The increased number of sweat gland-like structures observed in HESGCs cultured in media with FBS revealed that HESGCs had an increased proliferation capability compared with those cultured in media without FBS (Fig. 4C). RT-qPCR revealed that HESGCs cultured in media with FBS had higher expression levels of SG cell markers (CEA, K8, EDA and EDAR), whereas cells cultured in media without FBS expressed keratinocyte markers (K5, K14) at higher levels compared with those cultured with FBS (Fig. 4D).

In conclusion, serum is the primary factor that maintains the biological characteristics and functions of sweat gland cells, and medium without serum leads to the rapid differentiation of sweat gland cells into keratinocytes.

\section{Discussion}

The sweat gland is one of the important skin structures and its primary function is to regulate body temperature (22). The sweat gland cell is a somatic cell; therefore, its tolerance for in vitro amplification and subcultivation is low (23). The establishment of a novel culture method, which allows for the maintenance of the desired biological characteristics and improves sweat gland cell proliferation ability would be indispensable for future studies. The findings of the present study revealed that the two different types of media previously reported to be used for the culture of HESGCs had several shortcomings $(10,18)$. A serum-free medium, such as the KGM-2 medium, frequently led to sweat gland cells differentiating into keratinocytes, whereas a medium with serum, such as SG medium, reduced the HESGC 
proliferation ability. Based on the findings of the current study, a mix of SG medium and KGM-2 medium (SG:KGM 1:1 medium) may provide improved culture conditions for HESGCs.

The present study determined that HESGCs cultured in media without serum led to HESGCs rapidly differentiating into keratinocytes. HESGCs cultured in the mixed medium formed more sweat gland-like structures compared with cells cultured in the SG medium only. HESGCs cultured in the mixed medium also maintained the phenotypic characteristics of sweat gland cells, whereas cells cultured in KGM-2 medium did not. Overall, HESGCs cultured in the mixed medium maintained their biological characteristics and had increased proliferation ability. A previous study reported that HESGCs have the potential to generate epidermal keratinocytes that migrate toward local wounds in the epidermis (10). The findings of the present study were also consistent with the findings of previous studies which stated that when sweat gland cells were cultured in serum-free media, they rapidly lost their biological characteristics and differentiated into keratinocytes $(5,14)$.

Furthermore, the current findings suggested that media with serum allow for fibroblast survival and thus the maintenance of the HESGC biological characteristics; however, an excessive fibroblast population would have a competitive effect on HESGCs as fibroblasts have a higher proliferation ability. When the HESGCs were cultured in the SG medium only, a number of fibroblasts survived due to the high serum concentration, which led to the sweat gland cells being deprived of growth space. However, when the HESGCs were cultured in SG:KGM-2 (1:1) medium, moderate fibroblast growth allowed enough growth space for the sweat glands cells. Additionally, fibroblasts have the ability to secrete different types of factors, including basic fibroblast growth factor (bFGF), that stimulate cell growth (24), which is necessary for HESGCs and appropriate numbers of fibroblasts to coexist. The findings of the present study supported the hypothesis that media containing serum allowed for fibroblast survival and a fibroblast population may maintain the morphology and function of sweat gland cells. Several issues remain unsolved; including, if the fibroblasts are removed, it is uncertain whether or not the serum alone may directly maintain the characteristics of the sweat gland cells. It is also uncertain if the removal of the serum and the fibroblasts from the culture would allow the sweat gland cells to maintain their biological characteristics if the necessary factors such as TGF- $\beta$, PDGF, bFGF were added independently. Whether the serum directly maintained the biological characteristics of the sweat gland cells or allowed the fibroblasts and the HESGCs to coexist remains to be elucidated as some of the factors secreted by the fibroblasts that maintained the HESGC biological characteristics have not been identified.

In conclusion, the present study suggested that the coexistence and development of HESGCs with an appropriate number of fibroblasts may be considered in future HESGCs culture conditions. The use of mixed medium containing KGM-2 medium and SG medium may provide vital information for future sweat gland research. The mixed medium is a valuable compound and should be considered a future substantial supplemental medium.

\section{Acknowledgements}

We are grateful to Mr. YongPing Gu for histology. Funding for the present study was provided by the 973 Plan Research Special Subject Program (grant no. 2012CB22302), the National Natural Science Foundation (grant no. 81402584), the Natural Science Foundation of Jiangsu Province (grant no. BK20140360) and China National Textile and Apparel Council (grant no. J201405).

\section{References}

1. Cui CY and Schlessinger D: Eccrine sweat gland development and sweat secretion. Exp Dermatol 24: 644-650, 2015.

2. Dobson RL and Sato K: The secretion of salt and water by the eccrine weat gland. Arch Dermatol 105: 366-370, 1972.

3. Gallico GG III, O'Connor NE, Compton CC, Kehinde O and Green H: Permanent coverage of large burn wounds with autologous cultured human epithelium. N Engl J Med 311: 448-451, 1984.

4. Danner S, Kremer M, Petschnik AE, Nagel S, Zhang Z, Hopfner U, ReckhenrichG AK, Weber C, Schenck TL, Becker T, et al: The use of human sweat gland-derived stem cells for enhancing vascularization during dermal regeneration. J Invest Dermatol 132: 1707-1716, 2012.

5. Pontiggia L, Biedermann T, Böttcher-Haberzeth S, Oliveira C, Braziulis E, Klar AS, Meuli-Simmen C, Meuli M and Reichmann E: De novo epidermal regeneration using human eccrine sweat gland cells: Higher competence of secretory over absorptive cells. J Invest Dermatol 134: 1735-1742, 2014.

6. Li HH, Zhou G, Fu XB and Zhang L: Antigen expression of human eccrine sweat glands. J Cutan Pathol 36: 318-324, 2009.

7. Li HH, Fu XB, Zhang L and Zhou G: Comparison of proliferating cells between human adult and fetal eccrine sweat glands. Arch Dermatol Res 300: 173-176, 2008.

8. Hagmann S, Moradi B, Frank S, Dreher T, Kämmerer PW, Richter $\mathrm{W}$ and Gotterbarm T: Different culture media affect growth characteristics, surface marker distribution and chondrogenic differentiation of human bone marrow-derived mesenchymal stromal cells. BMC Musculoskelet Disord 14: 223, 2013.

9. Wang Y, Liu ZY, Zhao Q, Sun TZ, Ma K and Fu XB: Future application of hair follicle stem cells: Capable in differentiation into sweat gland cells. Chin Med J (Engl) 126: 3545-3552, 2013.

10. Böttcher-Haberzeth S, Biedermann T, Pontiggia L, Braziulis E, Schiestl C, Hendriks B, Eichhoff OM, Widmer DS, Meuli-Simmen C, Meuli M and Reichmann E: Human eccrine sweat gland cells turn into melanin-uptaking keratinocytes in dermo-epidermal skin substitutes. J Invest Dermatol 133: 316-324, 2013.

11. Schön M, Benwood J, O'Connell-Willstaedt T and Rheinwald JG: Human sweat gland myoepithelial cells express a unique set of cytokeratins and reveal the potential for alternative epithelial and mesenchymal differentiation states in culture. J Cell Sci 112: 1925-1936, 1999.

12. Czifra G, Szöllősi AG, Tóth BI, Demaude J, Bouez C, Breton L and Bíró T: Endocannabinoids regulate growth and survival of human eccrine sweat gland-derived epithelial cells. J Invest Dermatol 132: 1967-1976, 2012.

13. Lee CM, Carpenter F, Coaker T and Kealey T: The primary culture of epithelia from the secretory coil and collecting duct of normal human and cystic fibrotic eccrine sweat glands. J Cell Sci 83: 103-118, 1986.

14. Biedermann T, Pontiggia L, Böttcher-Haberzeth S, Tharakan S, Braziulis E, Schiestl C, Meuli M and Reichmann E: Human eccrine sweat gland cells can reconstitute a stratified epidermis. J Invest Dermatol 130: 1996-2009, 2010.

15. Lei X, Liu B, Wu J, Lu Y and Yang Y: Matrigel-induced tubular morphogenesis of human eccrine sweat gland epithelial cells. Anat Rec (Hoboken) 294: 1525-1531, 2011.

16. Tao R, Han Y, Chai J, Li D and Sun T: Isolation, culture, and verification of human sweat gland epithelial cells. Cytotechnology 62: 489-495, 2010.

17. Lei X, Wu J, Lu Y and Zhu T: Effects of acetylcholine chloride on intracellular calcium concentration of cultured sweat gland epithelial cells. Arch Dermatol Res 300: 335-341, 2008. 
18. Uchida N, Oura H, Nakanishi H, Urano Y and Arase S: Dispersed cell culture of human sweat duct cells under serum-free conditions. J Dermatol 20: 684-690, 1993.

19. Collie G, Buchwald M, Harper P and Riordan JR: Culture of sweat gland epithelial cells from normal individuals and patients with cystic fibrosis. In vitro Cell Dev Boil 21: 597-602, 1985.

20. Guenou H, Nissan X, Larcher F, Feteira J, Lemaitre G, Saidani M, Del Rio M, Barrault CC, Bernard FX, Peschanski M, et al: Human embryonic stem-cell derivatives for full reconstruction of the pluristratified epidermis: a preclinical study. Lancet 374 : $1745-1753,2009$

21. Sun Q, Li F, Li H, Chen RH, Gu YZ, Chen Y, Liang HS, You XR, Ding SS, Gao L, et al: Amniotic fluid stem cells provide considerable advantages in epidermal regeneration: $\mathrm{B} 7 \mathrm{H} 4$ creates a moderate inflammation microenvironment to promote wound repair. Sci Rep 5: 11560, 2015.
22. Lu C and Fuchs E: Sweat gland progenitors in development, homeostasis, and wound repair. Cold Spring Harb Perspect Med 4: pii: a015222, 2014.

23. Morimoto Y and Saga K: Proliferating cells in human eccrine and apocrine sweat glands. J Histochem Cytochem 43: 1217-1221, 1995.

24. Hainfellner JA, Voigtländer T, Ströbel T, Mazal PR, Maddalena AS, Aguzzi A and Budka H: Fibroblasts can express glial fibrillary acidic protein (GFAP) in vivo. J Neuropathol Exp Neurol 60: 449-461, 2001. 